from observational studies. Arthritis Rheum 2007; 56: 2896-2904.

4 Shakoor N, Michalska M, Harris CA, Block JA. Druginduced systemic lupus erythematosus associated with etanercept therapy. Lancet 2002; 359: 579-580.

5 Palucka AK, Blanck JP, Bennett L, Pascual V, Banchereau J. Cross-regulation of TNF and IFN- $\alpha$ in autoimmune diseases. Proc Natl Acad Sci USA 2005; 102: 3372-3377.

6 Bobbio-Pallavicini F, Alpini C, Caporali R, Avalle S, Bugatti S, Montecucco C. Autoantibody profile in rheumatoid arthritis during long-term infliximab treatment. Arthritis Res Ther 2004; 6: R264-R272.

7 Abunasser J, Forouhar FA, Metersky ML. Etanerceptinduced lupus erythematosus presenting as a unilateral pleural effusion. Chest 2008; 134: 850-863.
8 Hochberg MC. Updating the American College of Rheumatology revised criteria for the classification of systemic lupus erythematosus. Arthritis Rheum 1997; 40: 1725.

9 Madhusudan S, Foster M, Muthuramalingam SR, et al. A phase II study of etanercept (Enbrel), a tumor necrosis factor- $\alpha$ inhibitor in patients with metastatic breast cancer. Clin Cancer Res 2004; 10: 6528-6534.

$10 \mathrm{Abb} \mathrm{J}$, Kochen M, Deinhardt F. Interferon production in male homosexuals with the acquired immune deficiency syndrome (AIDS) or generalized lymphadenopathy. Infection 1984; 12: 240-242.

\title{
No lung cancer at resection of PET positive lesions: an audit of the lung cancer multidisciplinary team
}

\section{To the Editors:}

An essential role of the lung cancer multidisciplinary team (MDT) is to provide an accurate and prompt diagnosis enabling resection of malignant disease where appropriate, while minimising the risk of unnecessary surgical intervention. Since $2005,{ }^{18}$ F-deoxy-D-glucose positron emission tomography -computed tomography $\left({ }^{18}\right.$ FDG PET-CT) has been recommended for all patients with potentially radically treatable disease as part of the routine lung cancer staging process [1]. The Leeds Teaching Hospital (Leeds, UK) lung cancer MDT is a regional centre with an approximate workload of 500 patients with lung cancer per year. Within the lung cancer MDT, concern was raised regarding a series of patients referred for surgical resection who had a nonmalignant diagnosis at lung resection. It was decided to audit patients referred for surgical resection to determine the frequency of a benign diagnosis at resection. Standard practice within the lung cancer MDT is for patients to undergo thorough clinical assessment, followed by a contrast-enhanced CT scan of the chest and upper abdomen. ${ }^{18}$ FDG PET-CT scanning is selectively used if the patient may be suitable for potentially curative treatment. Depending upon the size and location of the suspected lung tumour, a tissue diagnosis is attempted via either fibreoptic bronchoscopy or CT-guided percutaneous needle biopsy.

All patients with a solitary pulmonary nodule presented at the lung cancer MDT were identified retrospectively from the lung cancer database between August 1, 2005, and August 30, 2008. The case records of the subset of patients who were referred for surgical resection were reviewed, and data pertaining to the investigations undertaken during the lung cancer diagnostic pathway and the final histological diagnosis at lung resection were obtained.

During the study period, 145 patients were referred for surgical resection. Of the patients, $52 \%$ were male and $48 \%$ were female. The median age of the patients was $69.5 \mathrm{yrs}$ (range 41.4-88.8 yrs). The most common diagnosis at resection was nonsmall cell carcinoma (table 1). Of the patients, $78(54 \%)$ had a solitary pulmonary nodule in which a tissue diagnosis could not be established prior to referral for surgical resection. All of these patients had received a thorough clinical assessment, and CT and ${ }^{18}$ FDG PET-CT scans. The opinion of the lung cancer MDT regarding these patients was that there was there was a high suspicion of a lung cancer based upon clinical and radiological assessment and, as a tissue diagnosis was not technically possible, surgical referral for resection was felt to be the appropriate course of action. Of the patients without a tissue diagnosis prior to surgical referral, $7.69 \%$

\section{TABLE 1 Diagnosis at surgical resection}

\begin{tabular}{lc} 
Diagnosis at surgical resection & Subjects \\
\hline Primary lung adenocarcinoma & $61(42)$ \\
Squamous carcinoma & $52(36)$ \\
Large cell carcinoma & $13(9)$ \\
Adenosquamous carcinoma & $3(2)$ \\
Large cell neuroendocrine tumour & $3(2)$ \\
Carcinoid tumour & $2(1.4)$ \\
Small and large cell carcinoma & $2(1.4)$ \\
Aspiration pneumonia & $1(0.7)$ \\
Chronic inflammation & $1(0.7)$ \\
Metastatic malignant melanoma & $1(0.7)$ \\
Metastatic colon carcinoma & $1(0.7)$ \\
Nonspecific inflammation & $1(0.7)$ \\
Pulmonary infarct secondary to Churg-Strauss & $1(0.7)$ \\
Small cell carcinoma & $1(0.7)$ \\
Tuberculoma & $1(0.7)$ \\
Abscess and organising pneumonia & $1(0.7)$
\end{tabular}


(95\% confidence interval 4.68-10.71) had a benign diagnosis following lung resection (table 1).

False-positive pulmonary ${ }^{18}$ FDG uptake is well recognised and causes include a wide range of infections and inflammatory conditions, which occur because these processes are associated with increased glucose (and glucose analogue) metabolism. There are now $>40$ benign conditions that have been reported to show increased ${ }^{18}$ FDG uptake [2]. In a meta-analysis of PET for diagnosis of pulmonary nodules and mass lesions, ${ }^{18}$ FDGPET was demonstrated to have a sensitivity and specificity for malignancy of 96.8 and $77.8 \%$, respectively [3].

The reduced specificity of ${ }^{18}$ FDG-PET may become more important over time as there is an increasing incidence of adenocarcinoma related to a shift to consumption of low-tar cigarettes [4]. Adenocarcinoma of the lung is the most common peripheral lung cancer; therefore, an increasing incidence may result in more solitary pulmonary nodules. Solitary pulmonary nodules can represent a diagnostic challenge to respiratory physicians and radiologists, as their peripheral nature and small size sometimes prevent a tissue diagnosis via either bronchoscopy or percutaneous CT-guided needle biopsy. There are a number of reasons why not all patients can have a histological diagnosis prior to lung resection, including position and site of the tumour, and comorbid risks, such as emphysema.

The present audit demonstrates that the risk of a benign diagnosis at surgical resection for patients with a solitary pulmonary nodule suspicious of lung cancer, where no tissue diagnosis has been established following clinical assessment and radiological assessment, and multidisciplinary team review is approximately $8 \%$. Patients need to be advised of the risk of a benign diagnosis prior to surgical resection so that they can make an informed decision regarding undertaking this treatment, which is not without a mortality risk. The search for a more specific positron emission tomography tracer continues.

\section{I.J. Clifton, A.F. Scarsbrook and M.F. Muers}

Leeds Teaching Hospitals NHS Trust, Leeds, UK.

\section{STATEMENT OF INTEREST}

None declared.

\section{REFERENCES}

1 NICE. The Diagnosis and Treatment of Lung Cancer. London, National Institute of Clinical Excellence, 2005.

2 Kavanagh PV, Stevenson AW, Chen MY, Clark PB. Nonneoplastic diseases in the chest showing increased activity on FDG PET. AJR Am J Roentgenol 2004; 183: 1133-1141.

3 Gould MK, Maclean CC, Kuschner WG, Rydzak CE, Owens DK. Accuracy of positron emission tomography for diagnosis of pulmonary nodules and mass lesions: a metaanalysis. JAMA 2001; 285: 914-924.

4 Janssen-Heijnen ML, Coebergh JW. The changing epidemiology of lung cancer in Europe. Lung Cancer 2003; 41: 245-258.

DOI: $10.1183 / 09031936.00176708$

\section{Smoking patterns in patients following a pulmonary embolism}

\section{To the Editors:}

Tobacco smoking is a major risk factor for cardiovascular disease. It has been found that $30-40 \%$ of patients stop smoking spontaneously after the occurrence of serious cardiovascular events [1]. Pulmonary embolism is a potential lethal cardiovascular event, in which smoking behaviour has not yet been documented. Since patients surviving the acute embolus face an increased risk of arterial thrombotic events, i.e. myocardial infarction and stroke [2-4], the propensity to quit smoking following pulmonary embolism is of particular interest.

Our objective was to determine the impact of an episode of pulmonary embolism on smoking behaviour. We compared the fraction of smokers and the percentage of successful smoking cessation in pulmonary embolism patients with those in population-based controls.

Data from a large follow-up project of patients with pulmonary embolism were used for the analysis. All patients who had experienced acute pulmonary embolism between January 2001 and July 2007 at the Leiden University Medical Center (Leiden, the Netherlands) were eligible. All patients were invited to a control visit in our clinic. An accurate smoking history was collected, including the smoking status at the time of the pulmonary embolism and smoking behaviour after the pulmonary embolism. Smoking statistics of population controls were derived from a nationwide registry on smoking habits in the Netherlands. By means of a continuous study by request of the Dutch government, 200 randomly selected Dutch households were contacted weekly to assess their smoking behaviour [5]. For the analysis, we used the data derived from the period 2001-2007 from population controls aged $>20 \mathrm{yrs}$, because of the limited fraction of pulmonary embolism patients aged $<20$ yrs $(0.3 \%)$.

A total of 675 patients were diagnosed with pulmonary embolism during the study period. Of the 675 patients, 225 $(33 \%)$ died before they could be questioned. Also, 139 patients of the remaining $450(31 \%)$ did not give informed consent for the follow-up visit because of comorbidity, geographic reasons, impossibility to give informed consent due to psychiatric 